\title{
An Implementation of Internet-connected Indoor Dust Bin Waste Level Monitoring System for Office Use
}

\author{
Mohammad Hafiz bin Ismail ${ }^{1 *}$, Tajul Rosli Razak ${ }^{2}$, Iman Hazwam Abd Halim³ \\ ${ }^{1,2,3}$ Faculty of Computer and Mathematical Sciences, Universiti Teknologi MARA, Perlis, Malaysia \\ Corresponding author:*MohammadHafiz@uitm.edu.my,mypapit@gmail.com
}

Received Date: 7 November 2019

Accepted Date: 11 November 2019

\begin{abstract}
The paper discusses an implementation of an internet-connected monitoring system to optimize waste collection within office space environment. The implementation consists of four parts - the physical dustbin module, network, middleware and user interface. The implementation uses NodeMCU board with embedded microcontroller to sense and transmit waste level data to a middleware over 802.11 wireless network, while the middleware aggregate data from all registered dustbins within the office area. The aggregated data is then stored in a database server for further analysis. The web-based user interface is used to monitor the dustbins waste level as well as historical data report. This information is vital for waste level management planning and prediction. Additionally, a Technology Acceptance Model survey result has shown that the monitoring system is perceived to be easy to use and useful for office management in monitoring and managing their waste.
\end{abstract}

Keywords: internet of things, smart dustbins, microcontroller, NodeMCU, esp8266, monitoring system

\section{INTRODUCTION}

Indoor dust bins designated for office spaces use usually consists of dry and solid waste which may include paper-based waste, plastic-based waste and stationaries. According to (United States Environmental Protection Agency, 2016), an average office worker generates an average of 2 pounds of paper and paperboard products waste every day and these wastes must be managed accordingly. Therefore, dust bins are usually placed nearby office workers' desk or on the floor where it is most convenient for workers to dispose of their refuse (Gollagher, Campbell \& Bremmer, 2017). The placement of the dustbins is also affected by the types of office space layouts.

Ideally, the waste from the dustbin would be collected once the bin is almost full and the garbage collector would proceed to collect waste from another dustbin. However, due to the lack of information on the waste level in each dustbin, the garbage collector would often find not every dustbin is filled with paper waste (Faccio, Persona \& Zanin, 2011). This creates a situation where the garbage collector must attend to every dustbin in the office floor regardless of their garbage level. This would result in inefficient use of garbage collection time in addition to loss productivity.

Thus, in this paper, we proposed an implementation of Internet-connected dustbin waste level monitoring system suitable for office space use. The monitoring system consists of three major parts: the embedded microcontroller on the dustbin, the middleware and the web-based user interface in remote server. 


\section{SYSTEM ARCHITECTURE}

The dustbin monitoring system is designed to be implemented within indoor environment and is designed to be fitted with existing commercially available dustbins to reduce operational costs. We have designed the system architecture according to the building blocks as suggested by Abdmeziem, Tandjaoui and Romdhani (2016). The overall monitoring system consists of four parts: Dust Bin module, Network Infrastructure, Middleware and User Interface

\section{Dust Bin Module}

The dustbin module is the on-site physical part of the system. The dust bin module is the first part of the monitoring system which is responsible for detecting the waste level in the bin and sending the information over the network. The dust bin component comprises of a sensing component, transmission component, microcontroller and a portable power supply as shown in Figure 1.

Sensing component uses HR-SC24 ultrasonic sensor in which the sensor is mounted at the top of the bin to measure the waste level relative the bottom of the bin by emitting high-frequency pulse and measuring the time taken for the echo to reflect at the sensor.

Transmission component is the part responsible for conveying sensor data to a remote receiver. The selection of transmission component is dependent on the transmission medium. As suggested by Zheng, Wang \& Wang (2015), several transmission mediums were considered for this project: raw ISM Radio frequency (433 MHz and $2.4 \mathrm{GHz}$ ), ZigBee and Wi-Fi (802.11). The factors considered for the transmission medium selection are implementation costs, effective communication range and ease of maintainability. For this project, Wi-Fi (802.11) was selected as almost all office spaces has Wi-Fi access point thus this will reduce complexity of deploying the system within office environment.

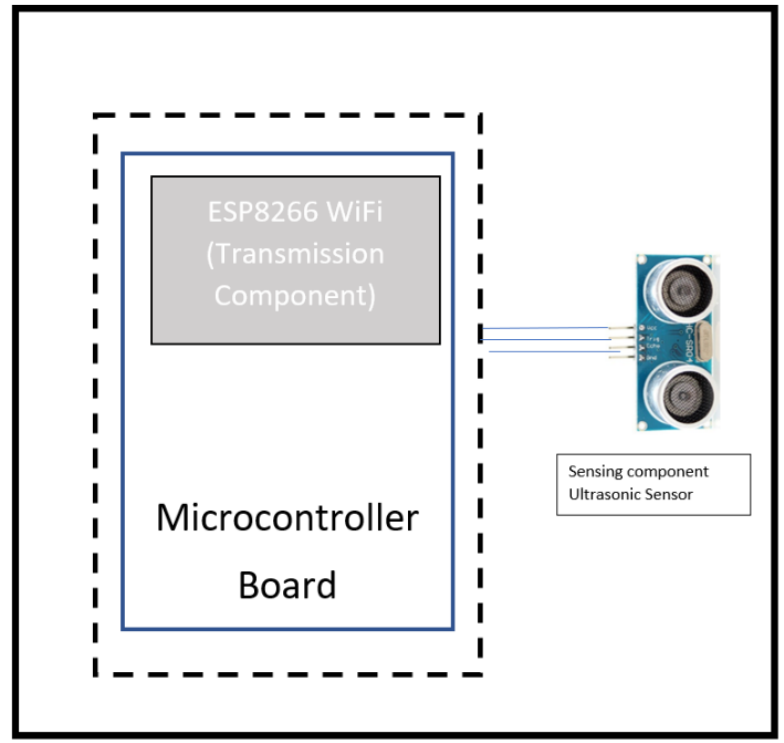

Figure 1: Dustbin Module

The dustbin module uses ESP8266 based NodeMCU V2 board to process input data from the ultrasonic sensor. This board has $32 \mathrm{bit}$ microprocessor running at $80 \mathrm{MHz}$ clock speed and comes with embedded 
TCP/IP which has built in Wi-Fi connectivity (Skraba, Kolozvari, et al, 2017). The compact design of the board made integration to the existing dustbin simpler. The primary function of the board is to determines the waste level based on ultrasonic result and sends out the data over the network.

The dustbin module is powered using Li-Ion battery with step up booster to convert 3.7-volt input from the battery to 5-volt output suitable for powering the microcontroller board and its sensors. To conserve battery power, the dustbin microcontroller is only set to read the sensor value at interval. This is done using deep sleep mode available in NodeMCU ESP8266 microcontroller board.

\section{Network Infrastructure and Middleware}

The monitoring system relies on 802.11 network infrastructure for communication between the dustbin module and its middleware. The middleware is written in PHP 7.1 and can be hosted either remotely or within the Local Area Network. The sensor data is transmitted from the dustbin module to the middleware using Hypertext Transfer Protocol (HTTP) GET method, where each data transmission contains the timestamp, dustbin id, Wi-Fi Access Point MAC Address, waste level sensor reading,

The middleware is responsible for aggregating sensor data from multiple dustbins and storing data in the database. The middleware will send trigger signal to monitoring client when the waste reached certain level. The overall block diagram is depicted in Figure 2.

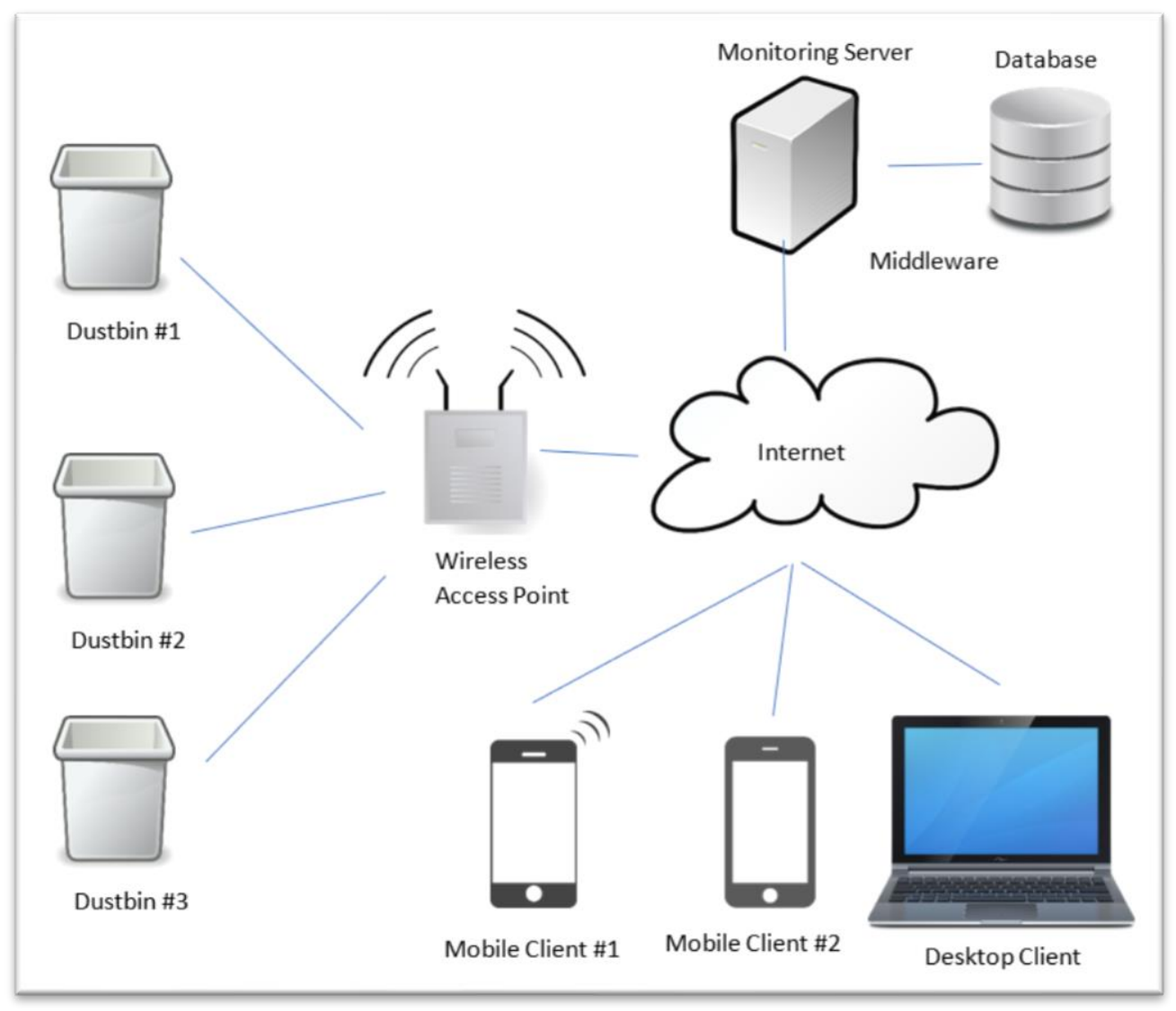

Figure 2: Block Diagram of Dustbin Monitoring System Network Communication 


\section{User Interface}

The user interface is the component which allow users to interact with the monitoring system. There are two user classes which can access the system, the Administrator and Normal Users (waste collectors). The bin administrator has full control over a registered bin. The administrator can add a new bin, updates, and deletes it. Remote bin with the embedded module will then transfer a bin data to the server. The administrators can also manage other users by modifying their account details.

Figure 3 is a screenshot of the monitoring system which display graphical representation of the waste bin level and it also store the historical data of waste level from waste bin.

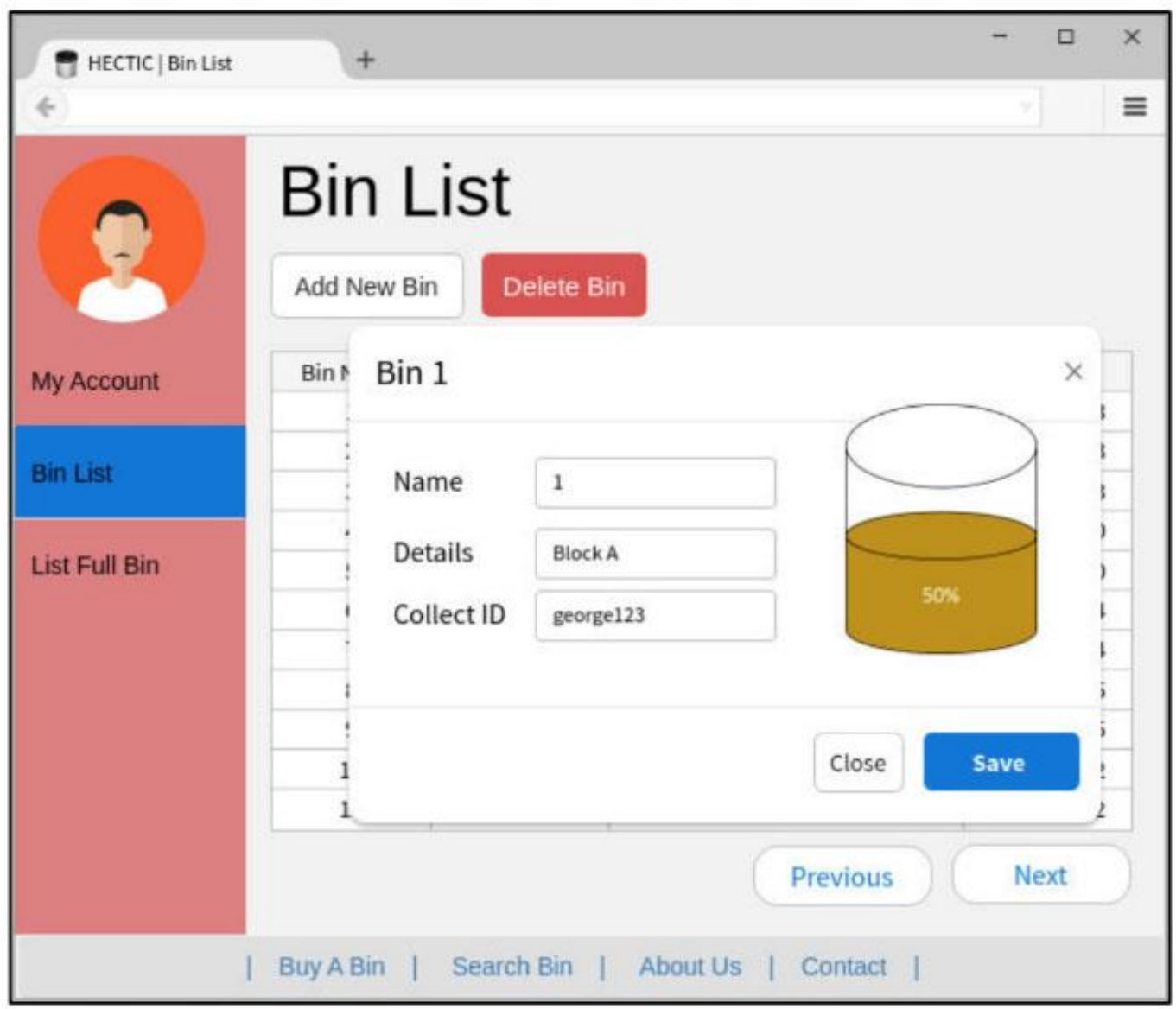

Figure 3: Waste Level Monitoring User Interface

The system can display historical graph of an individual bin waste level relative to their capacities in percentage. Figure 4 depicted the waste bin report screen. 


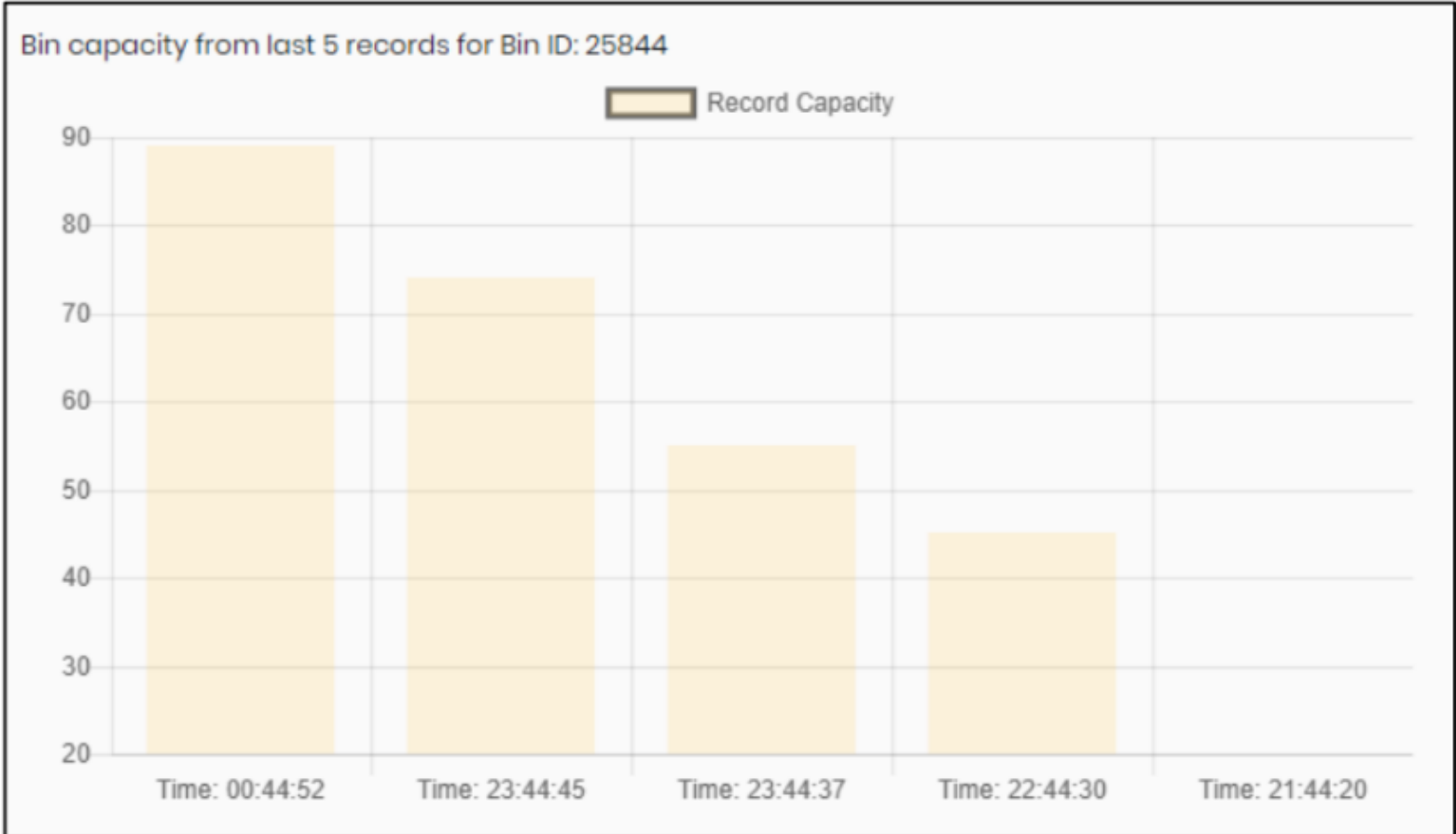

Figure 4: Dustbin Level Report Screen

\section{RESULTS AND DISCUSSIONS}

Burney and Ali (2017) conclude that some of the usability testing elements correlate with Technology Acceptance Model (TAM) factors and therefore its measuring systems are used to find the usefulness of the IoT-enabled dust bin. By studying the user's acceptance towards the system, some acknowledged problems can be solved to produce a more usable system environment. The TAM concepts influence each other in a way. Figure 5 depicts the relationships of these concepts.

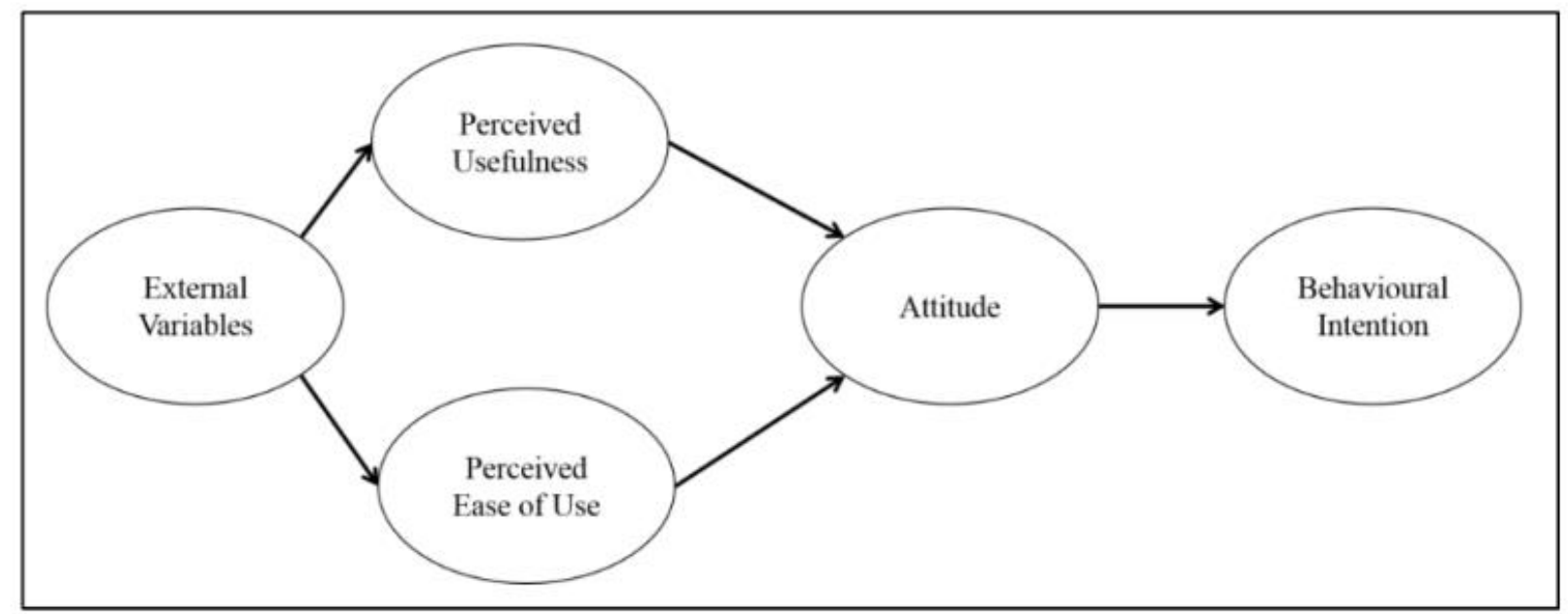

Figure 5: Technology Acceptance Model 
The potential users have been randomly chosen to test the dust bin monitoring system. Table 1 list the result of TAM survey results. Questions prepared are classified into four factors which are Perceived Ease of Use (PEOU), Attitude (AT), Perceived Usefulness (PU), and Behavioural Intentions (BI).

From PEOU criteria, the respondents find that the IoT enabled dustbin is easy to use and learning it is easy as well. Based on AT criteria, the respondents hold positive opinions in the IoT enabled dustbin while on PU criteria, the respondents think that the system is beneficial. On BI criteria, the respondents have the intentions to use the IoT enabled dustbin in the future.

Table 1: Result of Technology Acceptance Model Survey

\begin{tabular}{|l|l|c|c|c|}
\hline No. & Perceived Ease of Use Items & Min & Max & Mean \\
\hline Q1 & I find the IoT enabled Garbage Bin was easy to use & 3 & 5 & 4.07 \\
\hline Q2 & $\begin{array}{l}\text { Learning to use the IoT enabled Garbage Bin was easy for } \\
\text { me }\end{array}$ & 3 & 5 & 4.07 \\
\hline Q3 & The English language is not a barrier while using the system & 3 & 5 & 4.33 \\
\hline \multicolumn{5}{|l|}{} \\
\hline No. & Attitude Items & Min & Max & Mean \\
\hline Q4 & I have positive opinions in the IoT enabled Garbage Bin & 3 & 5 & 4.53 \\
\hline Q5 & $\begin{array}{l}\text { I think the usage of IoT enabled Garbage Bin is appropriate } \\
\text { for me }\end{array}$ & 3 & 5 & 4.33 \\
\hline No. & Perceived Usefulness Items & Min & Max & Mean \\
\hline Q6 & $\begin{array}{l}\text { The constructed system, as well as the bin, is more } \\
\text { convenient than other traditional garbage management }\end{array}$ & 3 & 5 & 4.13 \\
\hline Q7 & $\begin{array}{l}\text { IoT enabled Garbage Bin makes it easier to manage garbage } \\
\text { collection }\end{array}$ & 3 & 5 & 4.33 \\
\hline Q8 & $\begin{array}{l}\text { IoT enabled Garbage Bin improves my garbage management } \\
\text { skill }\end{array}$ & 3 & 5 & 4.07 \\
\hline Q9 & $\begin{array}{l}\text { IoT enabled Garbage Bin helps me manage garbage } \\
\text { collection efficiently }\end{array}$ & 3 & 5 & 4.33 \\
\hline Q10 & I think that IoT enabled Garbage Bin is useful & 3 & 5 & 4.53 \\
\hline
\end{tabular}

\section{CONCLUSION AND RECOMMENDATIONS}

Currently our dustbin implementation only focusses on dry offices' waste without regards of solid wet waste. Our current implementation also stresses more on the monitoring system rather than the microcontroller power efficiency. 
Future implementations of the indoor dustbin monitoring system should use a more compact ESP8266 microcontroller board instead of relying on NodeMCU development board which drains battery due to its integrated USB serial converter chip and the use of linear regulators.

The user interface for the dustbin monitoring system also needs improvement in order to make it more appealing to be used by consumers. It is also suggested that elements of gamification should be integrated in the waste level monitoring user interface as to provide incentives or encouragement to the users to reduce wastage and promote recycling behaviour.

\section{REFERENCES}

Abdmeziem, M. R., Tandjaoui, D., \& Romdhani, I. (2016). Architecting the internet of things: state of the art. In Robots and Sensor Clouds (pp. 55-75). Springer, Cham.

Burney, S. A., Ali, S. A., Ejaz, A., \& Siddiqui, F. A. (2017). Discovering the Correlation between Technology Acceptance Model and Usability. IJCSNS, 17(11), 53.

Faccio, M., Persona, A., \& Zanin, G. (2011). Waste collection multi objective model with real time traceability data. Waste management, 31(12), 2391-2405.

Gollagher, M., Campbell, J., \& Bremner, A. M. (2017). Collaboration Achieves Effective Waste Management Design at Brookfield Place Perth, Western Australia. Procedia engineering, 180, 17631772.

Škraba, A., Koložvari, A., Kofjač, D., Stojanović, R., Stanovov, V., \& Semenkin, E. (2017, June). Prototype of group heart rate monitoring with NODEMCU ESP8266. In 2017 6th Mediterranean Conference on Embedded Computing (MECO) (pp. 1-4). IEEE.

United States Environmental Protection Agency. (2016). "National Overview: Facts and Figures on Materials, Wastes and Recycling". Retrieved October 01, 2019, 2016, from https://www.epa.gov/facts-and-figures-about-materials-waste-and-recycling/national-overview-factsand-figures-materials

Zheng, D., Wang, Y., \& Wang, Y. (2015). Intelligent Monitoring System for Home Based on FRBF Neural Network. Int. J. Smart Home, 9(2), 207-218. 